\title{
New model of explanation for phase angle pattern of online partial discharge measurement in winding insulation of turbine generator
}

\begin{abstract}
Abdulrahman Baboraik ${ }^{1 *}$, Alexander Usachev ${ }^{1}$
On-line partial discharge (PD) measurement in electrical insulation of the turbine generator (TG) is an essential approach to control the quality of insulation and to avoid any undesired shutdowns of TG. Although in the last few decades the number of research in PD on-line monitoring methods in the stator winding of TG has increased significantly, it is still not clear yet why PD only appears at certain phase angles of the AC cycle in the phase resolved partial discharge (PRPD) pattern. Moreover, there is not yet any clarification on how the winding configuration of stator may affect PD phase angle pattern. For this reason, this work examines detailed study of the impact of the winding diagram on PD occurrence in the stator winding of real turbine generator class TVF-60-2 (60 MW, $10.5 \mathrm{KV})$. In addition, a computer simulation of various sizes of ellipsoidal cavities from 0.1 to $2.5 \mathrm{~mm}$ between conductor bar and stator core were carried out by using Laplaces equation in Finite Element Analysis (FEA) software ComSol to investigate the relation between the cavity size and coefficient of electric field. As a result of that, the phase angle of PD occurrence in the stator winding will highly depend on the configuration of bars connection, and the computer simulation has helped identifying the PD inception voltage and applied voltage of various ellipsoidal defects size. These two results have helped proposing an initial new theoretical model of explanation the relationship between voltage distributions and phase angle of PD occurrence to determine the degradation level of insulation caused by ellipsoidal defects in the insulation bars of the stator winding which can be used for stator windings of TG rated $6 \mathrm{kV}$ and higher. This model is the initial step to develop a further comprehensive model of explanation for PRPD patterns which will consider all other types of defects as well as the space charge effects from the previous PD.
\end{abstract}

K e y w ords: cavity, PD inception voltage, partial discharge, PD phase angle, stator winding

\section{Introduction}

Turbine generator is the main element of electric power systems and power plants. Therefore, monitoring of TGs operation conditions with low cost is a vital procedure to guarantee the operation reliability of power plants. However, breakdowns in the insulation of stator winding are the most frequent failures in TGs, which require more serious consideration during the monitoring process. Nowadays, online PD measurements is one of the most successful and widely used method for control and assessment of insulation condition in the stator windings of TG. This is because it minimizes the maintenance costs, reduces the interruptions, and increases the reliability of electrical services. In addition, the regular on-line measurements and the analysis of PD pattern during the operation dont only help in planning the essential maintenance during the normal outages of TG, but they also help in cost minimization of the downtime. These periodic on-line PD measurements are usually made once or twice a year to minimize the deterioration, which may be undetected between tests [1].

Although the number of $\mathrm{PD}$ research on developing the two most widely used PD interpretation approaches, including pulse height analysis (PHA) and PRPD, has increased significantly in the last few decades, the explanation of PRPD image is still a complex task, which requires more modeling and analysis [2]. Moreover, until now there is not any detailed explanation how can winding configuration of stator affects the $\mathrm{PD}$ pattern as well as $\mathrm{PD}$ phase angle, and how this relation can be used to determine the deterioration level in insulation. The consideration of winding configuration is only applied to identify in which slots the sensors should be placed in resistant temperature detector method [3] or to analyze partial discharge pulse propagation in the stator winding [4]. Even though there are some studies [5-6] in detecting the insulation deterioration in the stator winding, these studies do not consider the configuration of TG. Moreover, they do not have a uniform approach associated with the assessment of the accepted criteria of PD magnitude in the stator winding insulation of TG.

In the existing standards and manuals of online PD measurements in stator insulation of TG [7-8], two main characteristics of measured PD signals are magnitude and phase angles, which play a considerable role in analyzing PD pattern in the insulation of stator windings. However, using only PD magnitude without considering the nature and locations of PD is not reliable to evaluate

1 Kazan State Power Engineering University, Institute of Electric Power Engineering and Electronics, Department of Power Plant, 420066, Kazan City, Russian Federation, aleksandr_usachev@rambler.ru* Corresponding author: baboraik_4@mail.ru, kspeuamb@bk.ru 


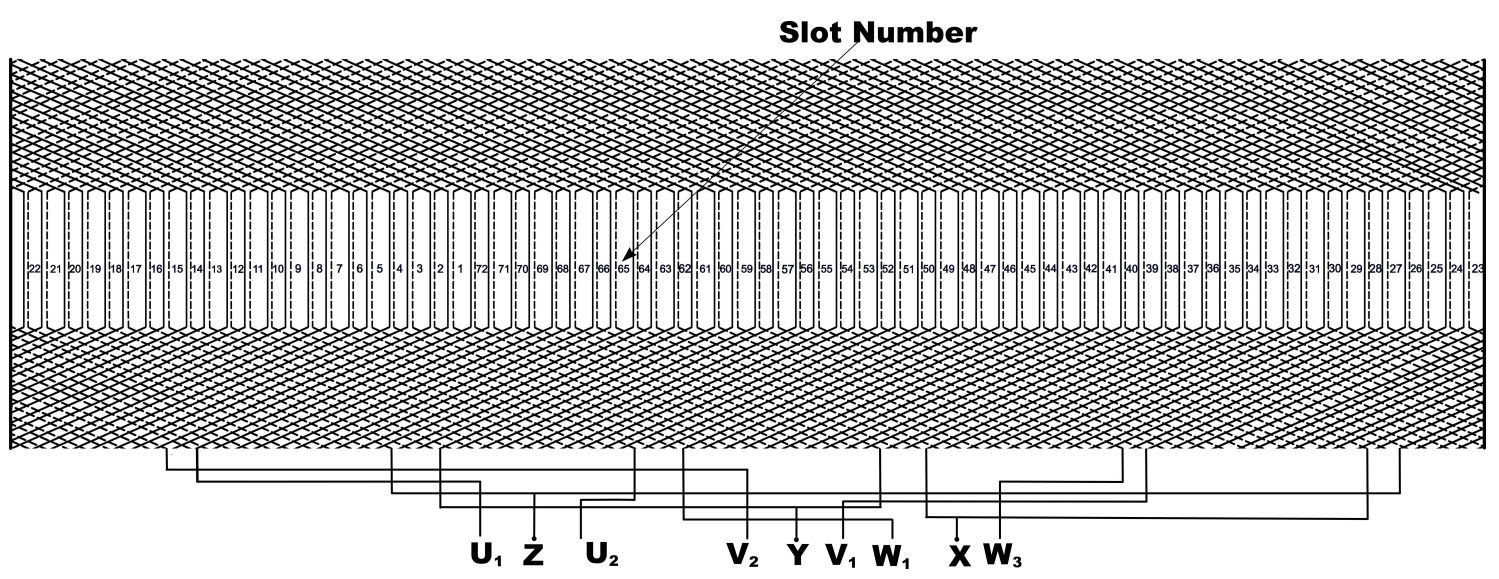

Fig. 1. Diagram of stator winding configuration of TG (TVF-60-2)

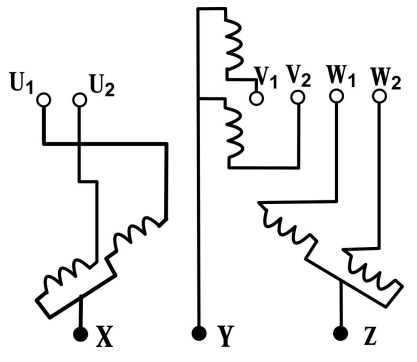

Fig. 2. Three-phase connection systems of TG

stator winding insulation [9]. Therefore, the phase angle is mainly used by many authors [10-12] to investigate the PD sources, such as slot, slot exit, and enwinding. However, the distribution of phase angle in the 2D and 3D plots is assessed differently in several research $[2,11,13-16]$, to locate the source of PD in the stator winding. These variations will be briefly introduced.

First, authors in [11,13-15] divide all PD in TG into two types:

1. Classic pulses: which occur in the slot part of the stator winding and they are either centered near $45^{\circ}$ pulses or $225^{\circ}$ phase angles of the alternating voltage.

2. Non-classic pulses: which occur in the endwinding area of the stator and they are centered at $15^{\circ}, 75^{\circ}, 195^{\circ}$ and $255^{\circ}$ phase angles of the alternating voltage. Furthermore, these authors in $[11,13]$ explain that the reason for this type of phase angle distributions of PD pulses is the fact that the insulation in the slot area is located under phase-to-ground voltage levels, while in the endwinding of the stator insulation is under phaseto-phase voltage.

On the other hand, in [16] it was said that if the detected PD are concentrated between $0^{\circ}-90^{\circ}$ and $180^{\circ}-$ $270^{\circ}$, they occur either in the slot area or slot exit. In contrast, if the PDs appear near $100^{\circ}$ and $280^{\circ}$, it means that PD occurs in the endwinding part between two different phases of the stator windings.
These significant differences in the authors' recommendations about the relationship between the phase angle of PD occurrence and the location of the defect is still a subject which raises many questions and riddles, which need to be investigated and answered, as many specialist still concerns about the reason of PD occurrence only at certain phase angles in PRPD pattern. In addition, all the previous mentioned works did not carry out any detailed investigations, which are based on real voltage distribution across the stator bars of the turbine generator or suggested a model to define the degradation levels of the insulation bar based on phase angle distributions.

Therefore, the main purpose of this paper is not only to investigate how stator winding configuration and voltage distribution in stator bars affect the phase angle of PD occurrence when the TG under real operating conditions but also to develop initial theoretical model to explain the PRPD patterns which can be used to define the degradation level of stator insulation.

\section{Research method}

The main used research method is the electrical detection method of PD measurement through the high voltage coupling capacitor (HVCC). In addition, the theory of gas discharges, the theory of electromagnetic induction, the mathematical statistics, the theory of long transmission lines, and the variational method of solving differential equations were applied in this study. Moreover, the finite element method of Laplaces equation solutions in the Comsol program was used to simulate the conditions of PD occurrence in the insulation of stator winding. The algorithm of interpretation PD phase angle and the analysis of relation between voltage distribution and stator winding configuration of the TG were carried out by using specialized developed programs DIWSGT created by authors in the Delphi environment. 


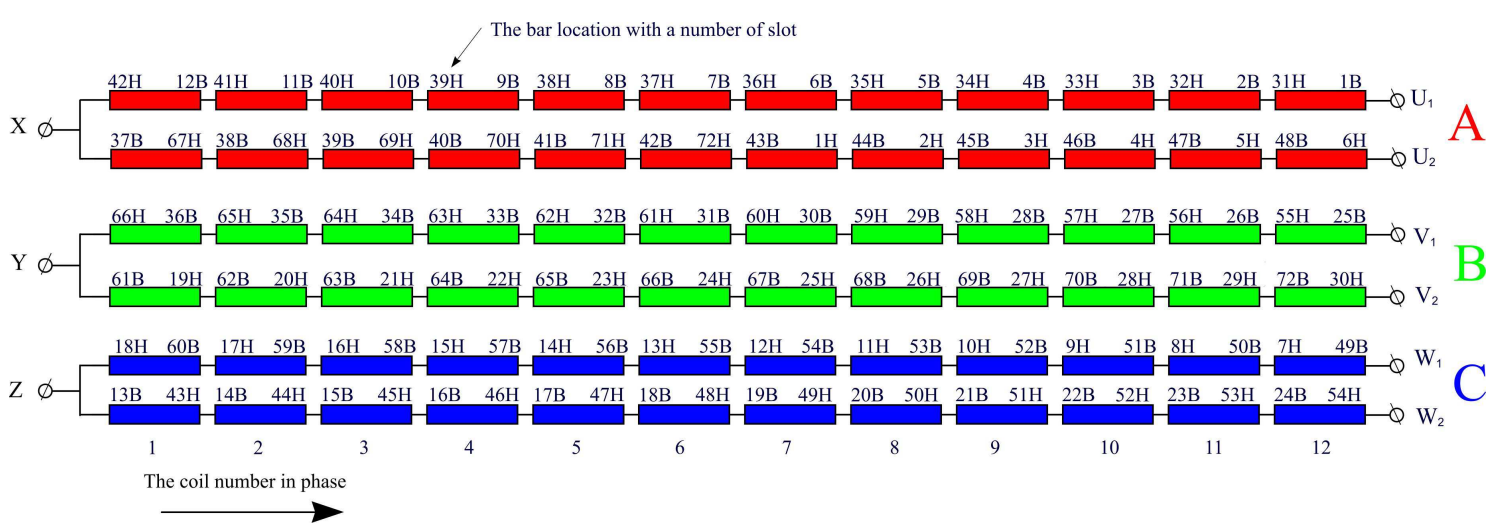

Fig. 3. Schematic view of numbering method of the bars

\section{The real condition of pd occurrence in the stator winding}

\subsection{The relation between voltage distribution and sta- tor winding configuration}

A turbine generator class TVF-60-2 (60 MW, 10.5 KV) was chosen to analyze the influence of voltage distribution on phase angle pattern of PD. The TG has the following specifications: terminal output voltage: $10 \mathrm{kV}$; stator winding cooling: hydrogen; stator current: $4330 \mathrm{~A}$; real power: $63 \mathrm{MW}$; stator winding: number of poles (p): 2 , number of slots per pole and phase (q): 24, number of parallel paths: 2 , number of bars in slot: 2 , pitch of winding: 28 , number of slots: 72 , total number of bars 144 $(3 \times 24 \times 2)$, slot length: $280 \mathrm{~cm}$, slot insulation type: MICA LMCH-P 0.17 thermoplastic insulation (asphalt mica), phase connection: star-star, number of bushings (terminals): 6 high voltage terminals, 3 neutral terminal.

First of all, the voltage distribution in bar-to-ground (phase-to-ground) in the stator winding of TG will be investigated based on the stator winding configuration to analyze the possibility of PD occurrence in any part of the stator winding. For this reason, the winding diagram of TG (TVF-60-2) was taken from the technical documents of existent power plants Kazan-TEC 2 Russia, Fig. 1. It may seem unserious to show this type of technical diagram; however, the common misunderstandings of PD occurrence conditions are caused by ignoring the detailed analysis of this winding diagram, which plays a significant role in clarification one of the most important related PD characteristics, PD inception voltage.

As it is difficult to deal with winding connection in this technical illustration Fig. 1, we will make representation of winding bars connection more obvious and elementary by representing them in the typical three-phase systems of TG Fig. 2 as well as in the schematic view of numbering method of stator bars Fig. 3. This in turn will simplify the understanding of voltage distribution in the stator winding.
In Fig. 2 and Fig. 3, the next symbols $U_{1}, U_{2}, V_{1}$, $V_{2}, W_{1}$, and $W_{2}$ indicate the high voltage terminals of phases $\mathrm{A}, \mathrm{B}$, and $\mathrm{C}$ respectively, whereas the symbols $\mathrm{X}$, $\mathrm{Y}$, and $\mathrm{Z}$ refer to the neutral terminals.

The numbering method of stator bars [17] Fig. 3 maps out which bars belong to each of the three phases, also it illustrates how bars and parallel circuits winding are connected within the single phase. In Fig. 3, each rectangle shows the location of coil (two series-connected bars), whereas the above shown symbol at the beginning and end of rectangular indicates the number of slot, in which this bar is located, while the letter refers to the location of bar in the slot ( $\mathrm{B}$ is for the top bar and $\mathrm{H}$ is for the bottom bar). For instance, the first rectangle in the right upper path of phase A is remarked $31 \mathrm{H} 1 \mathrm{~B}$. This means the $12^{\text {th }}$ coil in phase A consists of a top bar in $1^{\text {st }}$ slot, and it is connected to the bottom bar in $31^{\text {st }}$ slot.

According to above mentioned specification of TG the peak voltage in the each of 6 line terminals will equal $U_{0}=\frac{U_{\text {rat }} \sqrt{2}}{\sqrt{3}}=8.57 \mathrm{kV}$, if the TG has a symmetrical load and if the neutral points of the winding "star has zero potential. Moreover, the voltage for each bar will depend on its ordinal number, as every path of each phase consists of 24 series-connected bars, which often are sequentially numbered from neutral or high voltage terminals. For example, the bar located near to the neutral terminal will have zero potential at the beginning part and $U_{1}=\frac{U_{0}}{24}=$ $357 \mathrm{~V}$ at the end, whereas the $1^{\text {st }}$ bar located close to the high voltage terminal will have $U_{1}=\frac{U_{0}}{1} \cong 8.57 \mathrm{kV}$.

All the detailed explanations above may seem an unnecessary; however, they are very crucial in the next voltage distributions analysis, which helps to determine the value of partial discharge inception voltage as well as to investigate the real conditions of PD occurrence.

\subsection{The real conditions of PD occurrence in the slot area}

An analytic graphic software DIWSGT has been developed to calculate and figure out the voltage distribution for every single bar in clear and convenient way. This 


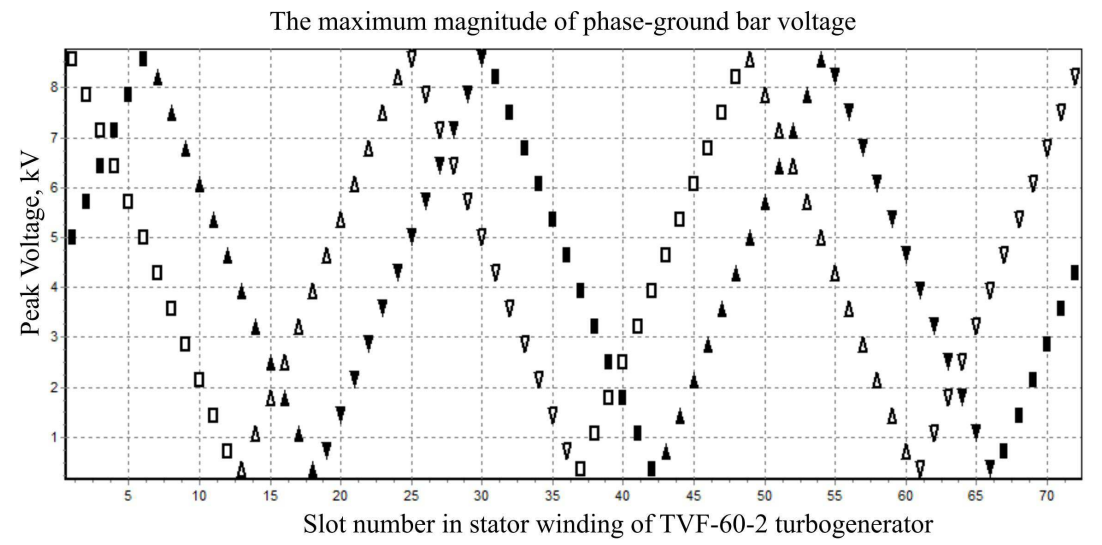

Fig. 4. Voltage distribution in the slot area of the stator bars in TG (TVF-60-2)

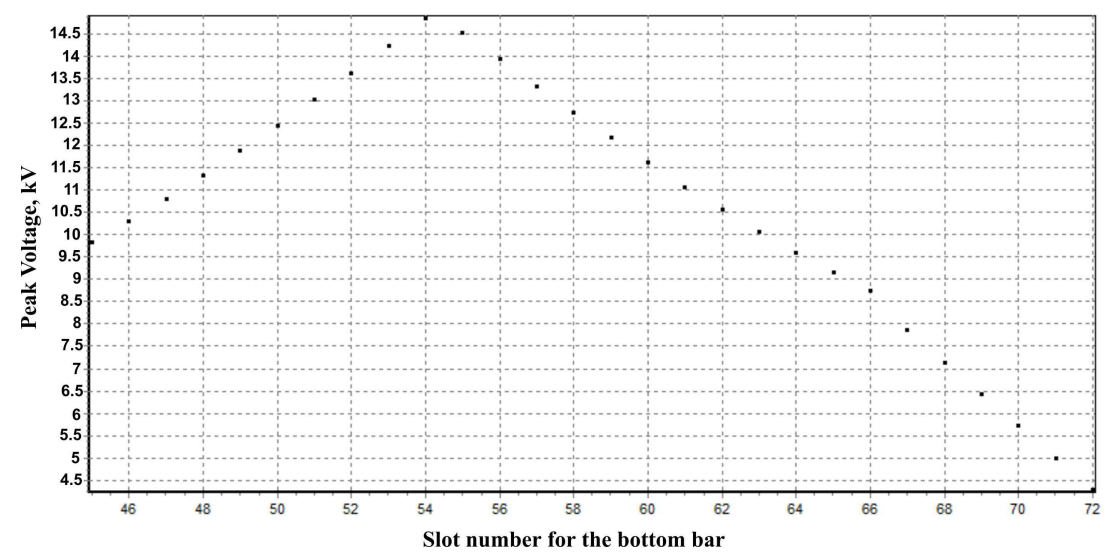

Fig. 5. Voltage distribution between top bar in the slot 1 and 28 bottom bars which cross it in enwinding area

software was officially registered in the state agency of software registrations in Russian Federation [18]. The required input data from TG specification are: type of phase connection, number of terminals, number of slots per pole and phase (q), number of parallel paths, number of bars in slot, and the pitch of winding.

According to the basic laws of electrical engineering, the voltage at the end of the any bar (number i) can be determined according to the formula (1), as the value of series connection bars, which are considered as the EMF sources as well as the amplitude value of the high voltage terminals at the beginning of the 1st bar in each phase are known:

$$
U_{i}=\frac{24-i}{24} U_{0}
$$

Where $i$ the bar number and $U_{i}$ the applied voltage on the bar number $i$. Therefore, the distribution of the maximum magnitude of phase-to-ground bar voltage in the slot area of the stator winding is illustrated by using DGWEST software, as shown in Fig. 4. The vertical symbols in Fig. 4 correspond to the voltage potential difference between the beginning and the end of the bar for all 144 bars which are located in the 72 slots.
Using the data in Fig. 4, it is evident that the bar-toground voltage for each bar in the same phase has different values; therefore, the conditions of PD occurrence in the bar insulation are different. For example, in slot number 1 there are two bars of phase A, the top one connected to the line terminal, which has the peak voltage $U \sim 8.57 \mathrm{kV}$, while the bottom one has phase-to-ground voltage $U \sim 5 \mathrm{kV}$. One more example, in slot 7 the bottom bar belongs to phase $\mathrm{C}(U \sim 8.2 \mathrm{kV})$ and the top one belongs to phase $\mathrm{A}(U \sim 4.2 \mathrm{kV})$. In addition, from Fig. 4 it can be seen that the maximum value of phaseto-ground voltage $(U \sim 8.57 \mathrm{kV})$ in stator winding can be obtained only in 6 bars, which are located in the slots number $1,6,25,30,49$, and 54, as they are connected to the high voltage terminals. In contrast, the six bars connected to the neutral terminals of the stator windings (in slots number 13, 18, 37, 42, 61, and 66) have phaseto-ground voltage equals to zero (or ranges between 0 to $360 \mathrm{~V}$ ). While, the bar-to-ground voltage in the each remaining 60 bars is different, and it ranges between 0.5 and $8.2 \mathrm{kV}$.

It is important to highlight that the phase-to-phase voltage in the slot area between two bars of different phases located in the same slot cannot be exist, because of 


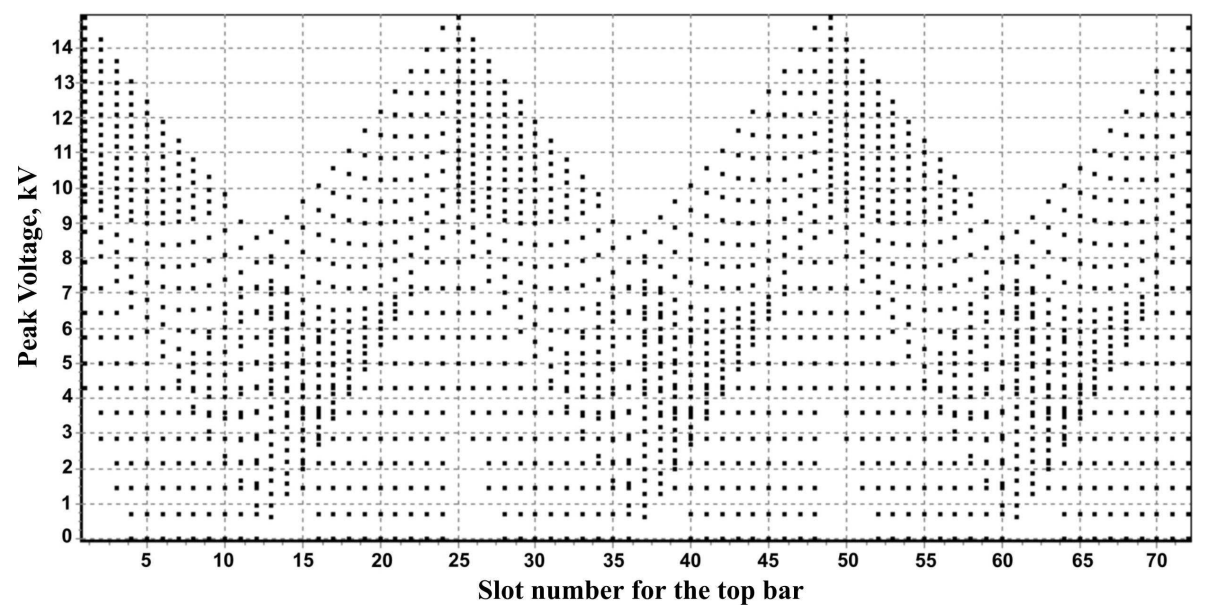

Fig. 6. The voltage between the top bars, crossing with the bottom bars from the other slots in enwinding area

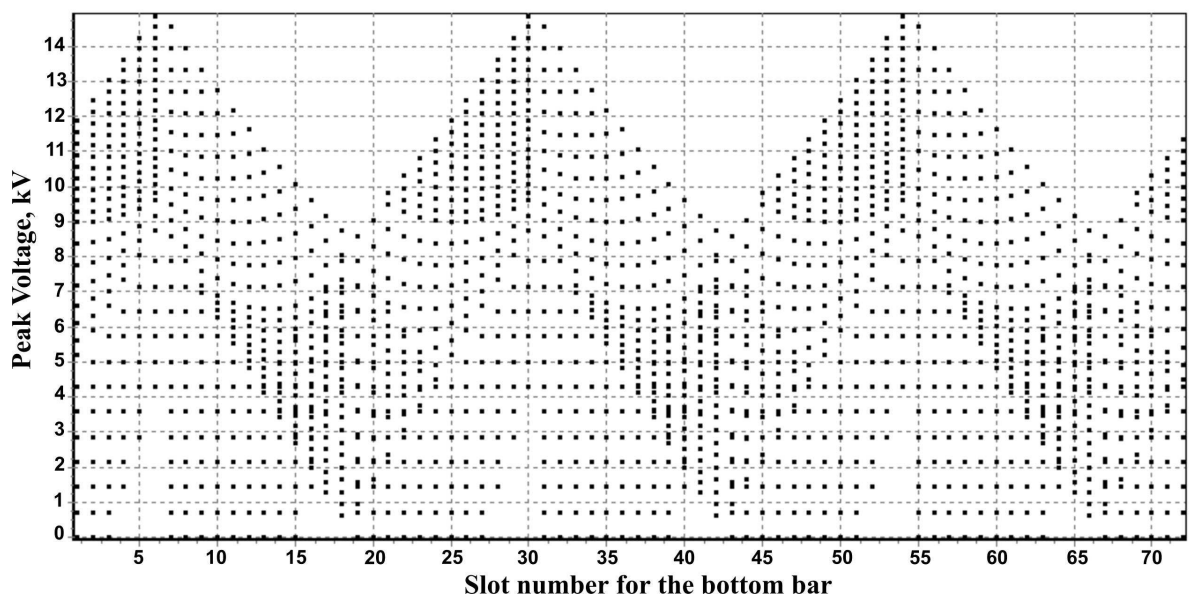

Fig. 7. The voltage between the bottom bars, crossing with the top bars from the other slots in enwinding area

the existence of semiconducting coating of MICA insulation between bars which approximately has $4 \mathrm{~mm}$ thickness, separator pad, and the strong ground-wall insulation which covers each bar [19-20].

Consequently, it is clear from the above analysis that the voltage between bar and ground differs in a wide range, and it highly depends on the order number of the bar in stator winding. Moreover, the possibility of PD occurrence is very weak in some bars, as the value of inception voltage is not enough to cause PD in stator insulation. Since the voltage in bars differs in a wide range the applied inception voltage and phase angle of PD will be also different. Then, PD cannot only appear near $45^{\circ}$ and $225^{\circ}$ phase angles of the alternating voltage as it was mentioned in $[11,12]$. This contradiction may affect the reliability of the PD phase angle which is a derived characteristic of the PD, as it is often considered as the main parameter to define the location of $\mathrm{PD}$. Therefore, a detailed investigation will be carried out in the remaining part of this work to make this point more obvious.
3.3 The real conditions of $P D$ occurrence in the endwinding area

According to the winding diagram of the TVF-60-2, in the end winding area each bar of the 144 bars crosses over either bottom or top other 28 bars which are coming out from the other stator slots. Consequently, there are $144 \times 28=4032$ crossing points in each side of the endwinding area. Therefore, the phase to phase voltage distribution between all crossing bars as well as the voltage between each bar and ground were determined in this work to investigate the condition of PD occurrence in the endwinding area Fig. 5. For instance, the Fig. 5 shows the voltage between the top bar exiting from the slot 1 and the bottom bars from the other 28 slots, which have crossing points with bar from slot 1 in the endwinding area.

It is evident from Fig. 5. that the maximum magnitude of phase to phase voltage $(14.85 \mathrm{kV})$ can be reached when the top bar in slot 1 crosses the bottom bar from slot 54 . The voltage distributions in the end winding area were calculated for each top bar in all 72 slots. As a consequence, the total number of graphs, which are similar to the graph shown in Fig. 5, will be 72 graphs. In addition, there are other 72 graphs is built for illustrating 
the voltage in crossing points between the bottom bars with other top bars in the endwinding. All 72 separated graphs for both of those combinations were combined into one graph to make the diagram of voltage distribution between bars in the endwinding area more clear, and they are shown in Fig. 6 and Fig. 7. Looking at these diagrams in more detail, it is evident that the maximum magnitude of phase to phase voltage $14.85 \mathrm{kV}$ was only at 3 crossing points in the endwinding area.

The description of voltage distributions at the crossing points of the bars in the endwinding area is illustrated on the vertical line with the corresponding slot number of the top and bottom bar in Fig. 6 and Fig. 7, respectively. In this case, the detailed information about which symbols of crossing points belong to a particular bar is lost. Because of this, the presented information in (Fig. 6 and Fig. 7) provides only a general view about the possible range of voltage magnitude in the endwinding area; moreover, the information about voltage at specific crossing points can be found by using graphical illustration type like the one shown in Fig. 5.

It is clear from Fig. 6 and Fig. 7 that there is a wide range of voltage ( 0 to $14.85 \mathrm{kV}$ ) between crossing bars in the endwinding area. Consequently, it is incorrect to say that the PD occurs in the endwindings only because of phase to phase voltage. Furthermore, there are some crossing points where there is high possibility of PD occurrence, such as in the crossing areas of top bars from slots number 1, 25, 49 (Fig. 6) and in the crossing area of bottom bars from the slots 6, 30, 54 (Fig. 7), while in other area the phase to phase voltage does not exist.

Since there is a large variation of voltage in the endwinding area, the question arises: why in [11-13] it was said that if the PD signals are located near $15^{\circ}, 75^{\circ}$, $195^{\circ}$ and $255^{\circ}$ phase angles of the alternating voltage, it means that the PDs occur in the endwinding area, as in this area the bars are located under the phase to phase voltage. This does not correspond to the current analysis of the voltage distribution in the stator winding, because the phase angles of PD occurrence will have a large variation. Therefore, the term "phase angle of PD occurrence becomes ambiguous and it cannot be represented by the $\mathrm{PD}$ inception voltage in the on-line PD test. This is because the peak value of the applied voltage $\left(U_{0}\right)$ in all parts of the test object (bars of stator winding) is not the same as in the off-line PD test, and the voltage in winding varies according to the law $U=U_{0} \sin (\omega t)$; consequently, the phase cycle at which the PD occurs is not particularly related to the inception voltage and it cannot be the exact characteristic of PD occurrence in online test comparing with the off-line PD test. Moreover, even if we determine phase angle during the PD measurements, it is impossible to determine the PD inception voltage, because the place of PD occurrence and the size of defect are not known. All the above investigations were essential to reach previous conclusion, as until the moment there is not any detailed explanation for voltage distribution in TG and their relation with PD occurrence voltage. Moreover, many specialists still concerns about the reason of $\mathrm{PD}$ occurrence at certain phase angles in PRPD pattern in the online test. Therefore, it was an essential to consider this basic knowledge to avoid making mistakes in using terminology during the interpretations of the results of PD measurements. For this reason, in the next part of this work we will answer the question how the phase angle can be used to diagnose the condition of insulation in the stator winding, as the phase angle cannot be only the parameter to determine the place of PD occurrence [19].

\section{Simulation of defect in the stator insulation}

First, to investigate the possibility of using the phase angle as the sign of insulation degradation in the stator winding, it is important to define the PD inception voltage based on the range of defects size, which may exist in the insulation of stator winding. This can be done through simulation by using the finite element method. Moreover, it is well known that to determine the value of the applied inception voltage $\left(U_{P D . i n c}\right)$, between the conductor bar and the ground, at which PD occurs in the defect (a gas cavity in insulation), it is necessary to know the values of the next parameters:

1. Distribution of the electric field in the defect and value of cavity inception voltage $\left(U_{b P D}\right)$.

2. Relation between electric field in the cavity and electric field in the remaining part of bar insulation where the cavity is not located. This relation will be called the average electric field next in this paper.

According to the Paschens law [20], if the electric field in the cavity is uniform, the applied inception voltage of cavity will depend on: the size cavity (b), dielectric material, temperature, pressure of the gas in the cavity, and the material of the electrodes. These parameters must be considered in the simulation in Finite Element Analysis (FEA) ComSol software.

The electrical discharge occurs in the gas cavity which is located between two electrodes separated by an insulating dielectric is called a barrier discharge. In addition, the magnitudes of the breakdown voltage $\left(E_{b P D}\right)$ as well as the inception voltage $\left(U_{b P D}\right)$ of the barrier discharge must be higher than the described values in Paschen's law, where the cavity is situated between metal electrodes, which are not separated by an insulating dielectric. This is because of the lower value of secondary ionization (Townsend second ionization coefficient $\gamma$ ) in the dielectric electrodes compared with a metal electrode. All these details will be taken into account in this simulation.

The value of cavity inception voltage $\left(U_{b P D}\right)$ for barrier discharge in the insulation of stator winding can be taken from [18]; however, we still need to find the distribution of the electric field and the average electric field in the defective part of insulation. Therefore, to determine these parameters, the range of possible sizes of ellipsoidal 


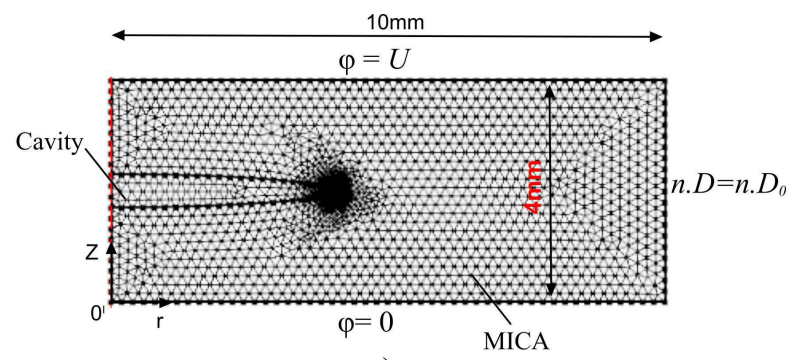

a)

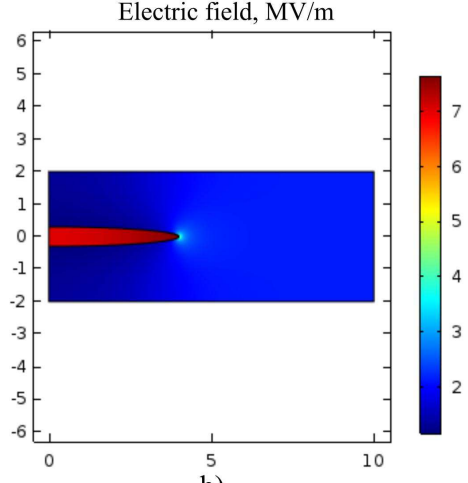

b)

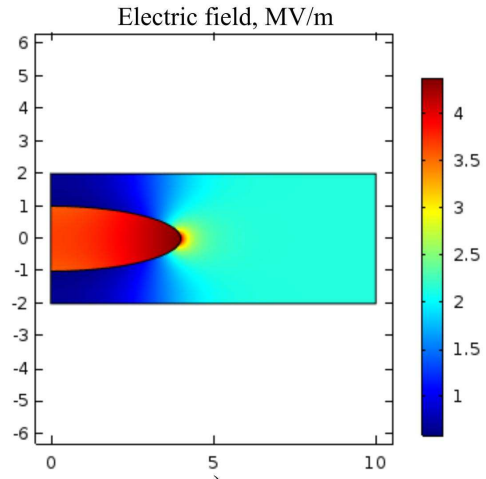

c)

Fig. 8. PD modelling: (a) - 2D axial-symmetric geometry, simulated electric field distribution in the MICA insulation with various defects: (b) $-0.6 \mathrm{~mm}$ and (c) $-2 \mathrm{~mm}$

Table 1. Definition of used parameters in the simulation

\begin{tabular}{lcc}
\hline Definition, symbol, unit & \multicolumn{2}{c}{ Value } \\
\hline Conductivity $\sigma, \mathrm{S} / \mathrm{m}$ & $2 \times 10^{-15}$ & $10^{-12}$ \\
Relative permittivity, $\varepsilon$ & 6 & 1 \\
Density $\rho, \mathrm{kg} / \mathrm{m}^{3}$ & 2900 & 1.2 \\
Thermal conductivity $\lambda, \mathrm{W} /(\mathrm{mK})$ & 0,5 & 0.024 \\
Specific heat capacity $\mathrm{C}, \mathrm{J} /(\mathrm{kgK})$ & 880 & 1.005 \\
\hline
\end{tabular}

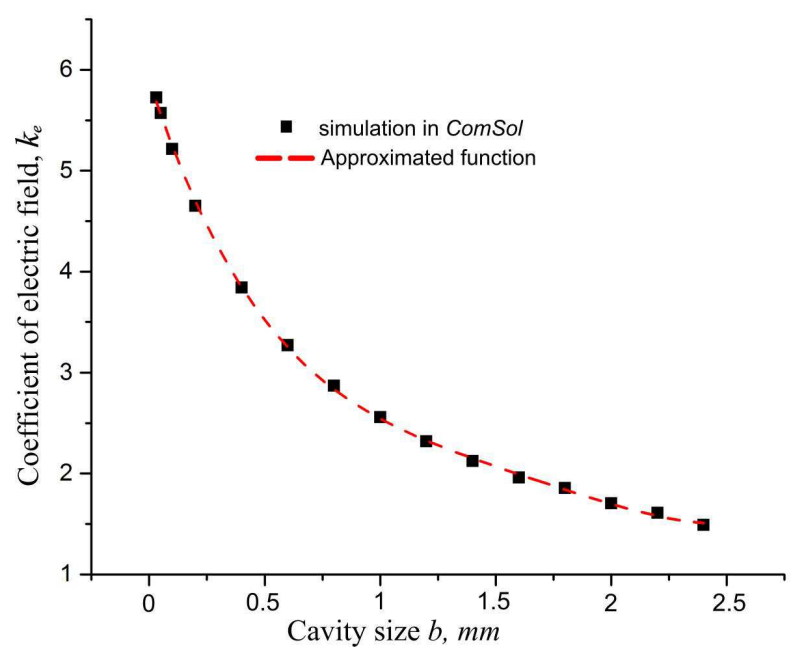

Fig. 9. Dependence of the coefficient of electric field on the cavity size

cavity in stator insulation of TG TVF-60-2 will be simulated by using ComSol software. The model of an elongated spheroid considers both type of internal PD: inside the insulation and between insulation and the stator core. However, the electric field inside cavity was only calcu- lated by Laplaces equation, and the effect of the space charge from previous PD (Poissons equation) has not been considered in this work, in addition, the other locations and shapes of cavity will be investigated in future research.

The two-dimensional (2D) axial-symmetric model geometry has been used in ComSol, as shown in Fig. 8. The model consists of MICA dielectric material $(\varepsilon=6.0)$ of $4 \mathrm{~mm}$ thickness and $10 \mathrm{~mm}$ diameter and an ellipsoidal cavity. A $50 \mathrm{~Hz}, 8.5 \mathrm{kV}$ AC sinusoidal applied voltage is applied to the upper electrode while the lower electrode is grounded. The main parameters of the simulation are shown in Table 1.

The results of the simulation of the electric field distributions in the MICA insulation with cavity in the stator bar are shown in Fig. 8.

It is obvious from the Fig. 8 that the electric field in a part of insulation, which is far from the defect, is uniform, and it almost has similar magnitude as any other point in the insulation. This electric field is called an average electric field (AEF) in bar insulation, and it equals the ratio of the applied voltage on the electrodes (thickness of the bar insulation). While the electric field inside and 
Table 2. Electric field distribution $E_{z}$ of ellipsoid cavities along the $r$ and $z$-axes of the model geometry in MICA insulation with different semi-axes $\frac{a}{b}$

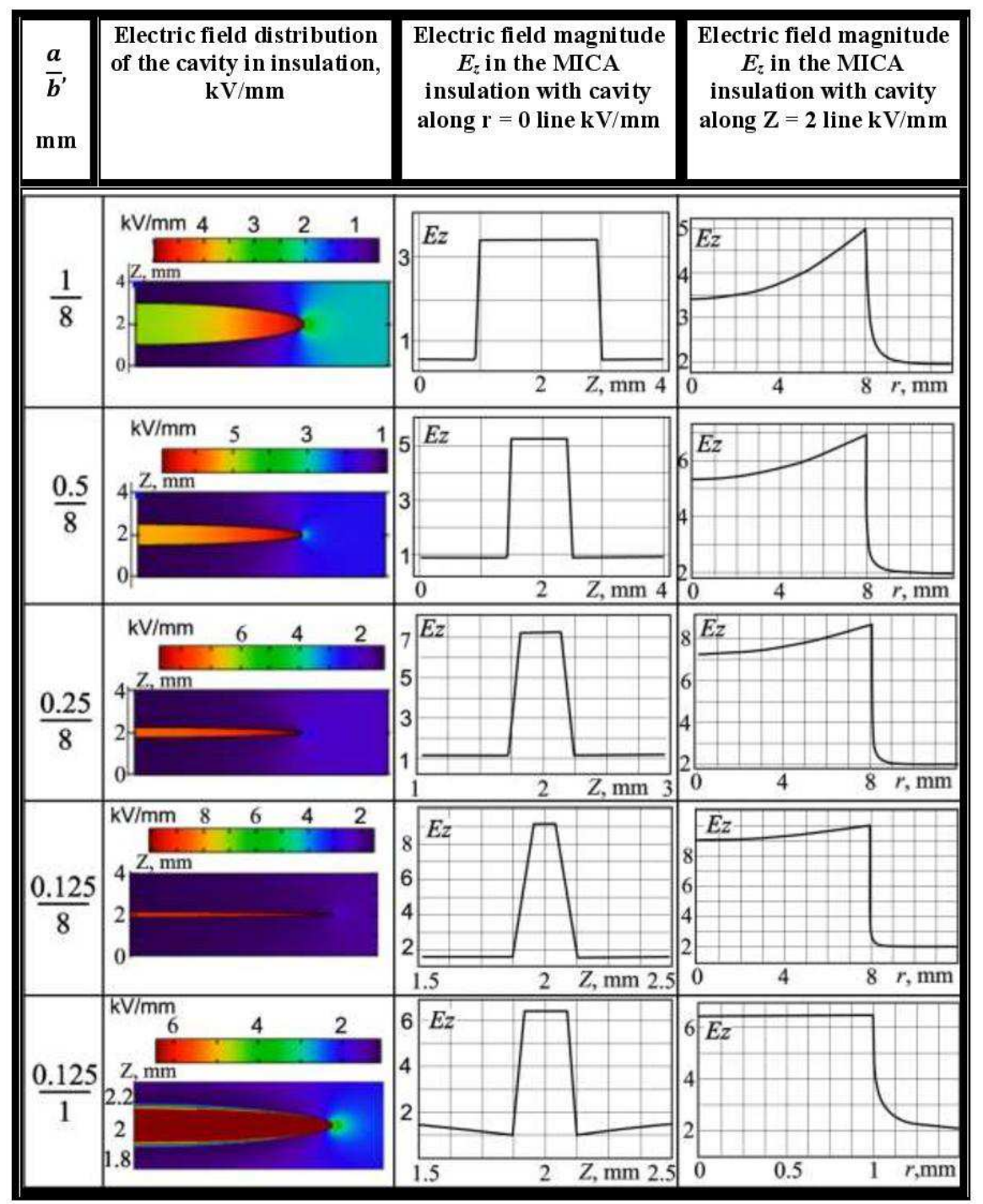

near the cavity differs from the magnitude of the AEF. A good illustration of this is that the electric field in the area of insulation above and below the cavity is less than the AEF in both defects in Fig. 8. Whereas, in the area of insulation, which is located close to the edge of the cavity, the magnitude of the electric field is higher than $\mathrm{AEF}$ and it may increase depending on the radius of curvature of the cavity shape. However, it is evident that the magnitude of the electric field inside the defect is greater than the average electric field in all other parts of the insulation bar.

Since the magnitude of the electric field is uniform for the most part of ellipsoidal cavity, the electric field distribution in the cavity cannot be symmetrical along the $r$ and $z$ axes of the model geometry. Because of this, more simulations of different cavities size were conducted and the results are shown in Table 2.

It is clear from Table 2 that the magnitude of the $E_{z}$ component of the electric field does not change along the $Z$-axis, as the electric field is uniform along the $Z$ axis except for the transition zone $(10-50 \mathrm{~nm})$ at the edges of the cavity. However, moving away from the axis of symmetry there is an observed increase in the $E_{z}$ which corresponds to an increase in the magnitude of the electric field in the cavity as its size decreases along $Z$ axis; consequently, the probability of PD occurrence is higher at the edges area of an ellipsoidal cavity.

Furthermore, it is clear from Fig. 8 and Table. 2 that the average of electric field approximately has the same magnitude which equals $2.14 \mathrm{MV} / \mathrm{m}$ (and $2 \mathrm{kV} / \mathrm{mm}$ in Table 2). The partial discharge in gas cavity occurs when the magnitude of the electric field reaches magnitude of inception voltage in the insulation. Moreover, the magnitude of the inception voltage depends on the size of the cavity. As can be seen from Table 2 when the size increases along the semi-axis $a$, the magnitude of the electric field inside the cavity decreased, while other parameters remained unchanged. In addition, the magnitude of 
the applied voltage does not play a fundamental role in defining relationship between the electric field inside and outside defect.

However, from an experimental point of view, to determine the magnitude of the applied voltage on the insulation of stator bar, at which a partial discharge occurs, it is necessary to know the ratio between the electric field in the cavity and the average value of the electric field in the part of bar insulation, where the cavity is located. Moreover, this ratio depends on the size of the cavity and it will be named as coefficient of electric field $\left(K_{e}\right)$.

Therefore, based on the simulation of electric field distributions in ellipsoidal cavities for various sizes (from 50 to $2400 \mu \mathrm{m}$ - common range size of defect along thickness of insulation in stator winding [21]) the dependence of the coefficient of the electric field $\left(K_{e}\right)$ on the size of the ellipsoidal cavity was calculated, as shown in Fig. 9. This dependence will help to determine how the cavity inception voltage will change when the cavity size in insulation also changes. Furthermore, a least squares approximation method was used to find the highest determination coefficient of approximated function and to find the $K_{e}$ for any cavity size:

$$
K_{e}=5.89-0.0069 b+5.4 b^{2}-2.1 b^{3}+3.19 b^{4} .
$$

The simulated and approximated results are depicted by the unfilled rectangular and dash line respectively.

All the previous practical calculations and simulations were important to find this function (2), which is crucial to find the needed cavity inception voltage and the applied voltage for any cavity size in the bar insulation of the stator winding. Moreover, it will be used to suggest a new initial model of explanation the phase angle of $\mathrm{PD}$.

\section{The model of explanation for the pattern of $\mathrm{PD}$ phase angle}

First of all, for explanation how the relationship between PD phase angle and defect size can be used to determine insulation degradation in the start winding, let us assume that we have a gas cavity $b=0.75 \mathrm{~mm}$ in the stator winding insulation of TG TVF-60-2 where the pressure of hydrogen is $p=200 \mathrm{kPa}$ and the insulation temperature equals $58^{\circ} \mathrm{C}$. In general, for this cavity size the value of AC applied inspection voltage can be determined according to Paschens law or graphs of experimental dependencies for the barrier discharge in $[22,23]$. Consequently, for this cavity $(b=0.75 \mathrm{~mm})$ the cavity inception voltage equals $U_{b P D}=3.3 \mathrm{kV}$. Further, the breakdown field strength can be obtained by determining the breakdown voltage for this cavity size which will equal $E_{b P D}=4.43 \mathrm{kV} / \mathrm{mm}$. To determine the value of applied inception voltage on the stator bar insulation for defect of $0.75 \mathrm{~mm}$, first of all we have to define coefficient of electric field $K_{e}$ for this cavity size by using the approximation function in Fig. 9. As a result, the cavity with $0.75 \mathrm{~mm}$ has coefficient equals $K_{e}=2.93$. This means that the PD only can occur in the gas cavity, if the average electric field in the bar insulation is 2.93 times less than normal. In this case, if the average electric field in bar insulation reaches $E_{\text {aver }}=1.51 \mathrm{MV} / \mathrm{m}$, which is calculated by the equation (3), then this value will be sufficient for the PD occurrence:

$$
E_{\text {aver }}=E_{b P D} / K_{e}
$$

Since the electric field in the bar insulation is uniform (from Table 2) and the insulation thickness is known $\left(b_{\text {insu }}=4 \mathrm{~mm}\right)$, the applied inception voltage $\left(U_{P D . i n c .}\right)$ will equal $6.04 \mathrm{kV}$, which is calculated by the next equation:

$$
U_{P D . i n c .}=E_{\text {aver }} b_{\text {insu }} .
$$

Further, the condition of PD occurrence in any bar can be described by the equation (5). The PD can be occur only if this applied voltage on the bar is equal or higher than PD inception voltage of the existent defect in this bar.

$$
U_{0 i} \geq U_{P D . i n c .} .
$$

Consequently, depending on which bar the cavity is located in, the PD will be observed at different values of the phase angle of the applied voltage. The Fig. 10 shows the values of phase angles, at which $\mathrm{PD}$ can be detected from the cavity with $b=0.75 \mathrm{~mm}$ and $U_{P D . i n c}=6.04 \mathrm{kV}$. These phase angles of $\mathrm{PD}$ is shown in every corresponding bar and slot of the stator winding of the TG TVF-60-2. And the symbols of the bars correspond to the symbols of Fig. 4, and the phase angle is measured from the 0 to 90 for all three phases A, B, and C.

It is clear from the results in Fig. 10 that if there are cavities with $0.75 \mathrm{~mm}$ size in the bars, which are situated in slots 11 to 20,35 to 44 and 59 to 68 , then the PD cannot be observed at any value of phase angle because in these slots the maximum voltage of bars is less than $U_{P D . i n c}<6 \mathrm{kV}$. In addition, the PD signals from similar size cavities, which are located in different bars, can be detected with different phase angle of the applied voltage, and the range of these phase angles varies from $44^{\circ}$ to $82^{\circ}$. Moreover, if the size of these cavities will increase from $0.75 \mathrm{~mm}$ to $1.1 \mathrm{~mm}$, the range of the $\mathrm{PD}$ phase angles will decrease to $60^{\circ}-90^{\circ}$ range, as shown in Fig. 11.

Therefore, it can be conclude that the phase angle of PD cannot be only the parameter to determine the cavity size and the place of PD occurrence in the online PD test in TG. However, how the phase angle of PD can be used to diagnose the condition of insulation in the stator winding?

To answer the previous question the next model is suggested. Initially, it is obvious from the relationship between PD phase angle and the defect size that the increase in size of ellipsoidal defect in the TG leads to the slow decrease in the breakdown voltage $\left(E_{b P D}\right)$ as well as in the coefficient of electric field $K_{E}$, while the applied 


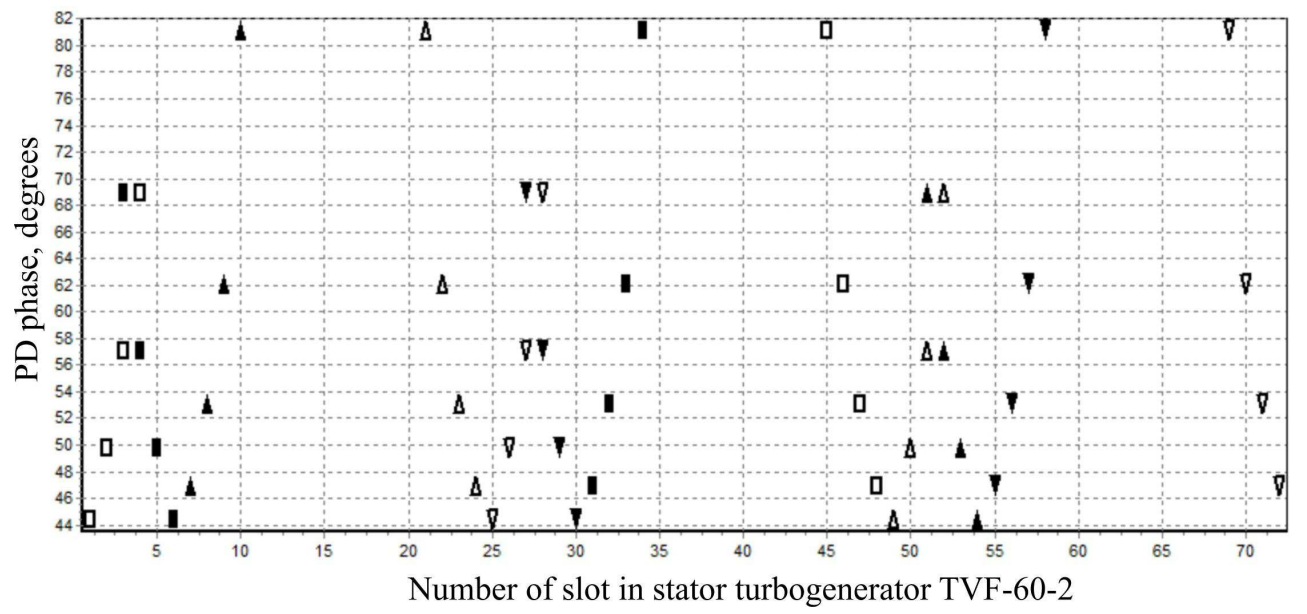

Fig. 10. The phase angle of the PD occurrence in the defect with $b=0.75 \mathrm{~mm}$

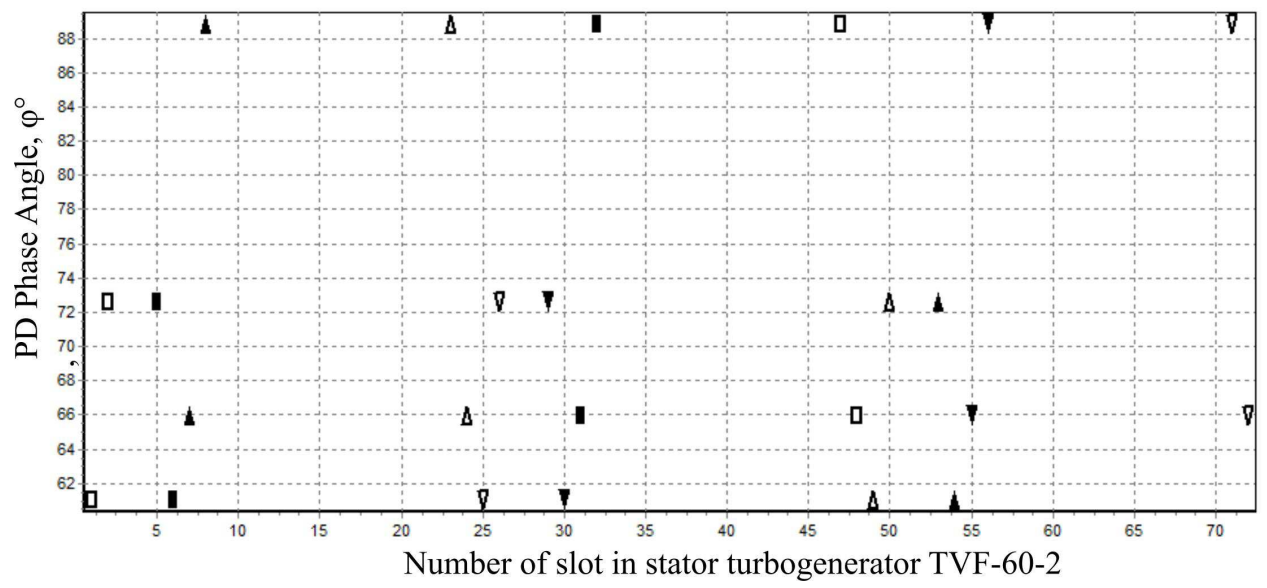

Fig. 11. The phase angle of the PD occurrence in the defect with $b=1.1 \mathrm{~mm}$ and $U_{P D . i n c .}=7.5 \mathrm{kV}$

inception voltage increases. This in turn leads to the increase in the phase angle of $\mathrm{PD}$ occurrence. Therefore, the next following model of explanation the PD phase angle pattern can be used to evaluate the condition of stator bar insulation: the trend of reduction in the range of phase angles can serve as a sign of an increase in the defects size of ellipsoidal defect as well as insulation degradation in the stator winding.

The trend of the PD phase angle pattern is the most helpful technique to classify the level of stator insulation deterioration. Moreover, a significant decrease in PD phase angle during a particular period will be a sign of critical stator insulation deterioration. The rate of considerable change in the range of the PD phase angle can serve as a key factor to determine if a warning should be given. The flowchart of this proposed model is shown in Fig. 12 .

This model can be used for analysis and explanation of PD phase angle pattern of any type of defect in the bar insulation for any class voltage of turbine generators.
However, this model is the first step of a future comprehensive model, which will incorporate space charge effects from the previous PD, and consider all other types of defects in the insulation of the turbine generators stator winding.

\section{Conclusions}

This paper analyzed the relation between the voltage distributions and the pattern of PD phase angle in the stator winding of TG model TVF-60-2, aiming to propose a new model of explanation PRPD pattern to determine the degradation level of stator insulation. The conducted analysis shows that the stator configuration of winding diagram considerably affects the conditions of PD occurrence and the phase angle of $\mathrm{PD}$, as the voltage varies according to the law $U=U_{0} \sin (\omega t)$ and the peak value of the applied voltage $\left(U_{0}\right)$ in all bars of stator winding is not the same as in the off-line PD test. Therefore, 


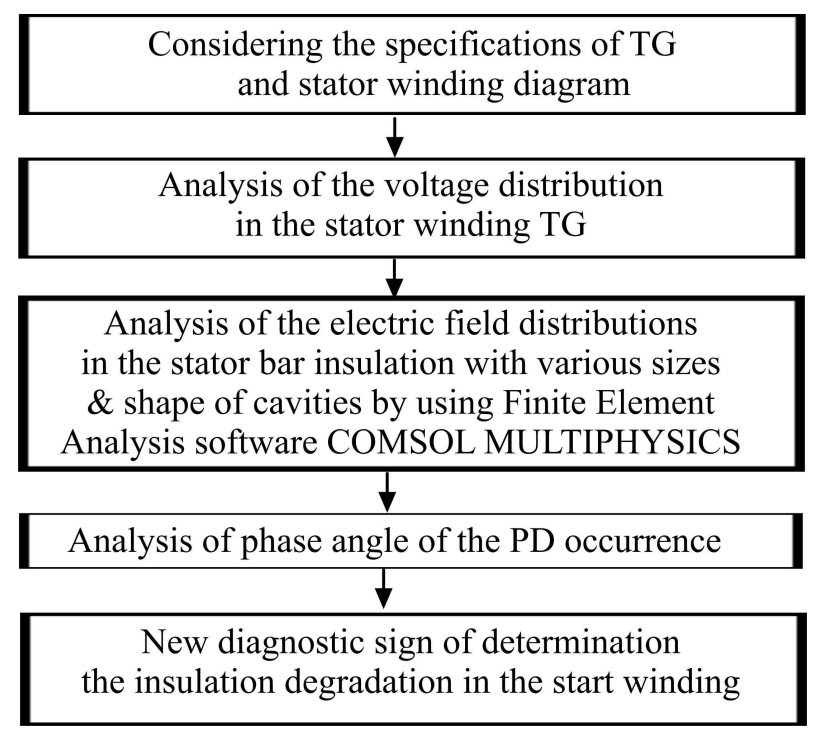

Fig. 12. The flow chart of proposed diagnostic model

there are some bars where the PD between the conductor bar and the ground cannot occur, while others have significant probability for PD occurrence. Moreover, the detected PD pulses from defects, which have similar sizes $0.7 \mathrm{~mm}$ and equal inception voltage $6.04 \mathrm{kV}$ but are located in different bars, should be observed at different values of the phase angle between $44^{\circ}$ and $82^{\circ}$. Consequently, the phase cycle at which PD occurs is not particularly related to the inception voltage of $\mathrm{PD}$ in the on-line PD test.

Furthermore, the simulation of ellipsoidal defects between conductor bar and ground in the stator insulation by using only Laplaces equation in FEA software ComSol has shown that the increase of defect size leads to the significant reduction of coefficient of electric field from 5.5 to 1.5 , which affects the pattern of the PD phase angle. As result of this dependence, the decrease in the range of phase angles of ellipsoidal defects, with $0.7 \mathrm{~mm}$ size in the internal part of bar insulation, from $44^{\circ}-82^{\circ}$ to $63^{\circ}-89^{\circ}$ can serve as a sign of their increase as well as insulation degradation in the stator winding of TG.

Based on the previous investigations, an initial new theoretical model of explanation PRPD pattern of ellipsoidal defects is proposed to diagnose the condition of stator bar insulation of turbine generators. Nevertheless, this model is the first step of a future comprehensive model, which will incorporate space charge effects from the previous $\mathrm{PD}$, and other types of defects in the insulation of the turbine generators stator winding.

\section{REFERENCES}

[1] IEEE Standards Association. IEEE Guide for the Measurement of Partial Discharges in AC Electric Machinery; IEEE Std $1434^{\mathrm{TM}}-2014$ (Revision of IEEE Std 1434-2000); IEEE Standards Association: Piscatawy, NJ,USA, 2014.
[2] Y. Luo, Z. Li, and H. Wang, "A Review of Online Partial Discharge Measurement of Large Generators",Energies,vol. 10, no. 11, p. 1694, 2017.

[3] W. Waluyo, S. Saodah, and E. Hidayatullah, "Internal On-line Partial Discharge Analysis of 68.75 MVA Generator Stator Winding Insulation",International Journal of Electrical and Computer Engineering, vol. 6, no. 5, p. 2088, 2016.

[4] M. Henriksen, G. C. Stone, and M. Kurtz, "Propagation of Partial Discharge and Noise Pulses in Turbine Generators", IEEE Transactions on Energy Conversion, vol. EC-1, no. 3, pp. 161-166, 1986.

[5] V. Warren, G. Stone, and H. Sedding, "Partial Discharge Testing: A Progress Report Stator Winding Insulation Thermal Class", Qualitrol - Iris Power, PD Progress Report IRMC, p. 17, 2017.

[6] Renforth L. et al, "A New Technique for the Remote Partial Discharge Monitoring of the Stator Insulation of High-Voltage Motors Located in Ex(hazardous) Locations", 2012 Petroleum and Chemical Industry Technical Conference (PCIC), IEEE, pp. 1-10, 2012.

[7] W. McDermid, "IEEE Guide for the Measurement of Partial Discharges in AC Electric Machinery", In2012 IEEE International Symposium on Electrical Insulation, IEEE, pp. 659-660, 2012.

[8] International Electrotechnical Commission. Rotating Electrical Machines-Part 27-2: On-Line Partial Discharge Measurements on the Stator Winding Insulation of Rotating Electrical Machines; IEC/TS 60034-27-2; International Electrotechnical Commission: Geneva, Switzerland, 2012.

[9] H. Zhu, V. Green, D. Huynh, and L. Ravenscroft, "How to Identify Stator Insulation Problems using On-Line Partial Discharge Analysis", 2000 IEEE International Symposium on Electrical Insulation Cat. No. 00CH37075, pp. 506-511, 2000.

10] G.C. Stone, I. Culbert, E.A. Boulter, H. Dhirani, "Electrical Insulation for Rotating Machines: Design, Evaluation, Aging, Testing, and Repair, 2nd ed.; John Wiley \& Sons Inc.: Piscataway, NJ, USA, pp. 159-234, 2014.

[11] V. A. Rusov, "Measurement of Partial Discharges in the Insulation of High-Voltage Electrical Equipment", Ekaterinburg, USURT, p. 367, 2011.

12] M. Hoof, S. Lanz, "PD Diagnostics on Rotating Machines. Possibilities and Limitations", Proceedings: Electrical Insulation Conference and Electrical Manufacturing and Coil Winding Conference (Cat. No. 99CH37035), IEEE, pp. 195-200, 1999.

13] M. Kurtz, J. F. Lyies, G. C. Stone, "Application of Partial Discharge Testing to Hydro Generator Maintenance, IEEE Transactions On Power Apparatus and Systems., vol. PAS-103, no. 8, pp. 195-200, 1984.

[14] LLC Dimrus, "Guidelines for the Organization of Partial Discharges Measurement in the Insulation of the Stators of HighVoltage Electrical Machines", Perm: LLC Dimrus, p. 14, 2013.

[15] Iris QMS, "Interpretation of PD Results - On-Line Testing Version 3.2", Iris QMS, ver. 3.2, 2010.

[16] J. M. Pemen, "Detection of Partial Discharges in Stator Windings of Turbine Generators", Eindhoven, Technische Universität, 2000 .

17] Klempner, Geoff, and Isidor Kerszenbaum, "Handbook of Large Turbo-Generator Operation and Maintenance", 2nd ed., Wiley-IEEE Press, p. 1032, 2018.

[18] A. M. Baboraik, A. E. Usachev, "The State Registration of the Computer Program DIWSGT for the Determination Degradation of Insulation in the Stator Winding of Operating Turbogenerators by the Partial Discharge Method", Federal Institute of Industrial Property (FIPS), No. 2018662196, 2018.

19] A. M. Baboraik, A. E. Usachev, Z. M. Ali, "A New Proposed Method for Interpretation On-Line Partial Discharge Measurements Data of Turbine Generator", J. Electr. Eng. Technol, no. 15 , pp. 1301-1311, 2020. 
[20] J. Kuffel, P. Kuffel, "High Voltage Engineering Fundamentals", Newnes, 2000.

[21] A. M. Baboraik, A.E. Usachev, "Features of the Partial Discharges Detection in the Stator Winding of Turbine-Generators in Online and Offline Modes", Twenty-Third International Scientific and Technical Conference of Students and Postgraduates Students Radio Electronics, Electrical Engineering and Power Engineering: Materials of Reports, Moscow: MEI, vol. 3, p. 400, 2017.

[22] H. A. Illias, G. Chen, P. L. Lewin, Modelling of Partial Discharge Activity in Different Spherical Cavity Sizes and Locations within a Dielectric Insulation Material", In2009 IEEE 9th International Conference on the Properties and Applications of Dielectric Materials, IEEE, pp. 485-488, 2009.

[23] G. A. Mesetes, "The Laws of Similarity in Pulsed Gas Discharges UFN. Reviews of Actual Problems", vol. 176, no. 10, pp. 1069-1091, 2006.

Received 11 September 2020

A. M. Baboraik graduated from Kazan State Power Engineering University (KSPEU), Kazan, Russian Federation $(\mathrm{RF})$, in 2011 with a bachelors degree with honors in electrical power engineering- power plants and substations. Then, he received Msc degree with honors in electrical power engineeringrenewable energy in 2013 from the same university. He worked as a lecturer and primary instructor (2014-2015) and research associate (2013-2017) in the department of power plant at KSPEU. In 2018, he earned not only a PhD degree in diagnostics insulation of high-voltage electrical equipment but also a degree of teacher-researcher qualification in photonics, instrumentation, optical and biotechnical systems and technologies from KSPEU. Currently works as academic researcher \& consultant in renewable energy, and member in research laboratory of Modeling, Analysis and Control of Systems (MACS) in KSPEU. His research interests include diagnostics insulation of high-voltage electrical equipment, and electricity generation from renewable energy sources (solar photovoltaic, solar thermal, wind, and geothermal).

A. E. Usachev was born in Russia in 1951, graduated from faculty of physics at Kazan State University, Soviet Russia, in 1973 with a higher education diploma in radiophysics and electronics. From 1973 until 1996, he worked as a senior laboratory assistant, senior laboratory engineer, junior research assistant, research associate, and senior research associate at Kazan Institute of Physics and Technology of the Sciences Academy of Soviet Russia as well as the Russian Federation (RF). He obtained PhD in solid-state physics (condensed matter physics) from Kazan Institute of Physics and Technology, Soviet Russia in 1982. He has been working since 1996 until the present at the Kazan State Power Engineering University at the department of power plants as associate professor (19962000) and professor (since 2000 until today). He received a post-doctoral degree in physical and mathematical sciences, from Kazan Institute of Physics and Technology Kazan, RF in 2000. He worked as a dean of the Electric Power University in 2000, Kazan, RF. His research interests include: high-voltage electrical engineering, diagnostics insulation of high-voltage electrical equipment and lightning protection. 